\title{
Patrones geográficos de distribución de árboles y arbustos en la zona de transición climática mediterráneo-templada de Chile
}

\author{
Geographic patterns of distribution of trees and shrubs in the transitional \\ Mediterranean-temperate climatic zone of Chile
}

\author{
ERNESTO A. TENEB, LOHENGRIN A. CAVIERES*, MARÍA JOSÉ PARRA \& ALICIA MARTICORENA \\ Departamento de Botánica, Facultad de Ciencias Naturales y Oceanográficas, \\ Universidad de Concepción, Casilla 160-C, Concepción, Chile \\ *autor para correspondencia: email: lcaviere@udec.cl
}

\begin{abstract}
RESUMEN
Uno de los principales objetivos de la biogeografía es determinar si las especies se distribuyen en el espacio en forma aleatoria o si existen grupos de especies con patrones de distribución comunes. En el presente estudio determinamos a través de técnicas cuantitativas si existen corotipos, es decir, grupos de especies con patrones de distribución común entre los árboles y arbustos en la zona de transición climática mediterráneotemplada de Chile, Octava Región de Chile. Adicionalmente, evaluamos la relación entre el clima y la distribución de los diferentes grupos de árboles y arbustos de la Octava Región. El análisis de agrupamiento de especies permitió definir 24 corotipos significativos, de los cuales 18 responden significativamente con alguna variable ambiental analizada. La temperatura media anual, la temperatura media invernal y la temperatura media de verano resultaron ser las variables que mejor explican el patrón de distribución de la mayoría de los corotipos. Las tendencias geográficas de los corotipos y su relación con las variables ambientales fueron evaluadas mediante un análisis de ordenación. Los resultados señalan que los corotipos con distribución en la cordillera de los Andes se correlacionan con la temperatura de invierno en tanto que los corotipos con distribución costera se correlacionan con la mediterraneidad. Se discute respecto de la utilización de una nueva aproximación para el análisis fitogeográfico y de su utilidad en el estudio de la relación entre la vegetación y los factores ambientales que determinan su distribución.
\end{abstract}

Palabras clave: corología, corotipo, variables ambientales, fitogeografía, transición climática.

\begin{abstract}
One main aim of biogeography is to determine if species are randomly distributed or if there exist groups of species with common distributional patterns known as chorotypes. In this study, we determined through quantitative techniques if there exists such chorotypes for trees and shrubs in the transitional Mediterraneantemperate climatic zone of Chile, Eigth Administrative Region of Chile. Additionally, we evaluated the relationship between climate and distribution of the different chorotypes. The analyses allowed defining 24 significant chorotypes, 18 of which were significantly associated with some used environmental variable. Mean annual temperature, mean summer temperature and mean winter temperature were the variables that explained the distribution of most of the chorotypes. The geographic tendencies of chorotypes and their relationship with environmental variables were evaluated with canonical correspondence analysis. The results indicated that chorotypes distributed in the Andes Range were correlated with the mean winter temperature, whereas chorotypes with coastal distribution were correlated with mediterreanity. We discusses the use of new approaches for phytogeographic analysis and its utility in the study of the relationship between vegetation and environmental factors that determine its distribution.
\end{abstract}

Key words: chorology, chorotype, environmental variables, phytogeography, climatic transition.

\section{INTRODUCCIÓN}

Una de las principales metas de la biogeografía es detectar si las especies se distribuyen independientemente unas de otras, o si por el contrario existen patrones de distribución común entre varias especies (Brown \& Lomolino 1998). Si efectivamente existen patrones de distribución común a varias especies, entonces estas son susceptibles a ser clasificadas definiendo límites más o menos claros entre un grupo de especies y otro (Real et al. 1992a, 
1992b, 1997, Márquez et al. 1997, Vargas et al. 1997). De este modo, se consigue la simplificación de complejos patrones de distribución individual por patrones de distribución de grupos de especies, permitiendo a su vez relacionarlos con factores ambientales.

En el análisis de la distribución de especies existen dos grandes aproximaciones: (1) detectar patrones biogeográficos repetitivos (e.g., grupos de taxa con distribuciones similares) y (2) detectar regiones bióticas (e.g., grupos de áreas con biotas similares). Dentro de la primera aproximación está la búsqueda de corotipos, los que han sido definidos por Birks (1987) como un grupo de taxa con distribución similar (Real et al. 1992b). Vargas et al. (1997), precisando la definición anterior, sugiere definir corotipo como un grupo de especies cuya superposición en sus distribuciones es mayor a la esperada por azar.

Las zonas de transición climática ofrecen un atractivo particular para los análisis biogeográficos (Morla \& Pineda 1985), esto porque el sola- pamiento entre tipos climáticos distintos crea gradientes ambientales que repercuten en la flora y que pueden ser analizados considerando la riqueza, composición, recambio y diversidad de especies, pudiendo relacionarlos a su vez con factores ambientales (Blasi et al. 1999). Sin embargo, se tiene un escaso conocimiento de la ecología y biogeografía de las zonas de transición climática, lo que se debe a la escasa proporción de terreno que ocupan con respecto a las regiones climáticamente homogéneas y con vegetación invariable (Blasi et al. 1999).

La flora de Chile central posee un especial interés para los biogeógrafos dada la gran diversidad de ecosistemas que presenta, destacándose la Octava Región por sus peculiaridades geográficas, climáticas y florísticas (Cavieres et al. 2004). Esta región es considerada como una zona de transición entre los climas mediterráneo y templado, cambio que está determinado por una variación en los regímenes pluviométricos con aumento de las precipitaciones de norte a sur (Di Castri \& Hajek 1976). Como

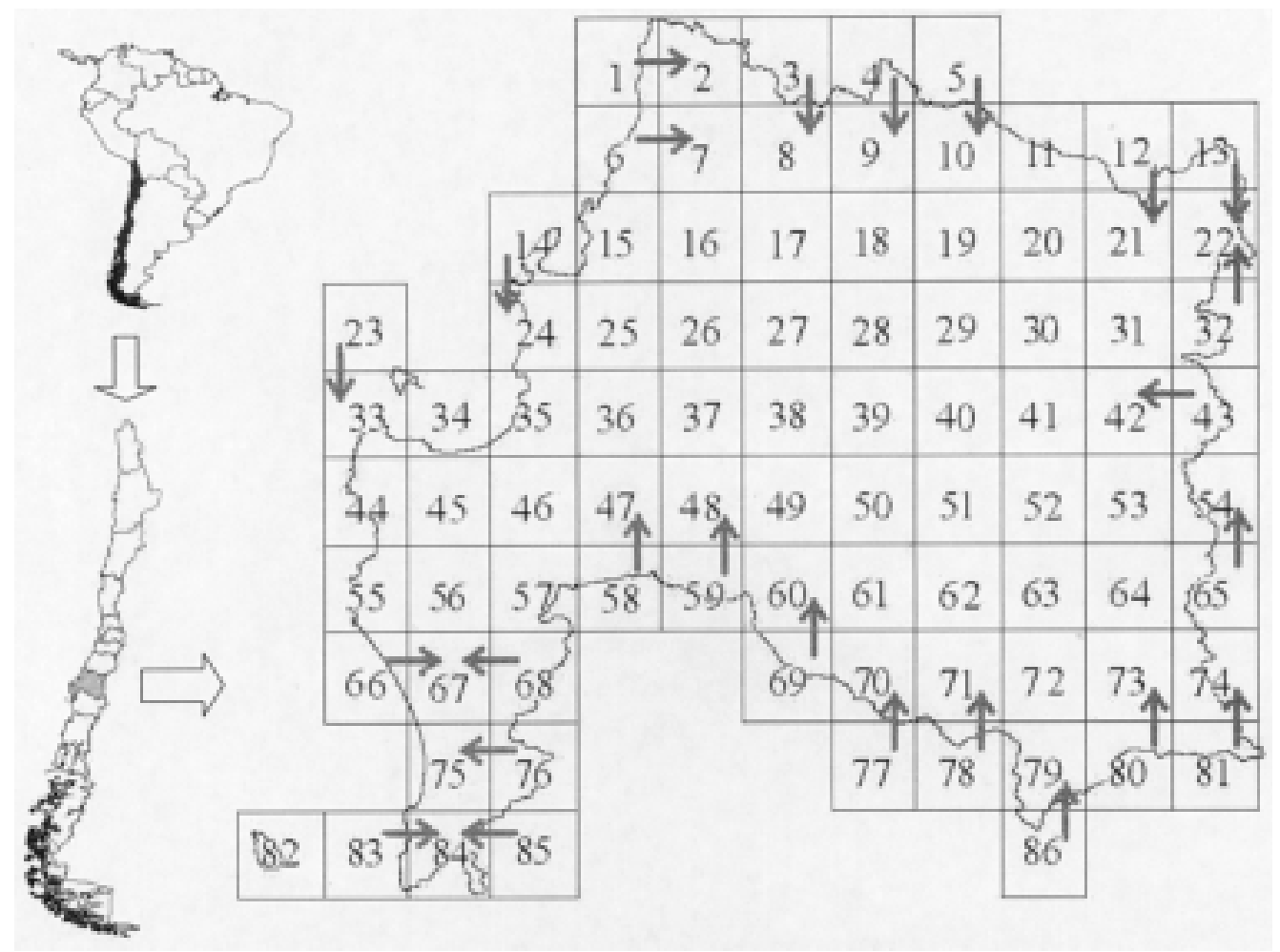

Fig. 1: Ubicación geográfica de la Octava Región de Chile mostrando la grilla de 0,25 x 0,25 grados de latitud-longitud usada en este estudio. También se indican con flechas las cuadrículas que fueron fusionadas.

Geographic location of Eigth Region of Chile showing the grid of 0.25 x 0.25 degrees latitude-longitude used in this study. Arrows indicate the quadrats that were fused. 
consecuencia de lo anterior, la Octava Región es considerada como una zona de contacto entre la flora esclerófila típica del clima mediterráneo y la flora correspondiente al bosque templado lluvioso, exhibiendo alta riqueza de especies (Cavieres et al. 2004).

La Octava Región ha sido estudiada principalmente desde una perspectiva vegetacional, y dentro de análisis más extensos que han abarcado todo el país (Pisano 1956, Schmithüsen 1956, Oberdorfer 1960, Huber 1975, Gajardo 1994). Sin embargo, hasta ahora, no existen estudios de índole florísticos donde se analicen los patrones geográficos de distribución de especies dentro de la región. No obstante, las asociaciones vegetales encontradas en los trabajos fitosociológicos han sido representadas en mapas que aportan una idea de lo que podemos encontrar en un análisis fitogeográfico. Sin embargo, se hace necesario validar estas propuestas fitogeográficas con análisis corológicos que complementen la información ya existente. Además, la definición de corotipos permitiría relacionar los patrones encontrados con variables ambientales, pasando de una fase descriptiva a una fase generadora de hipótesis (Real et al. 1992b).

Los objetivos de este trabajo son: (1) determinar entre árboles y arbustos presentes en la Octava Región grupos de especies que posean patrones de distribución significativamente similar, y (2) determinar factores ambientales que están correlacionados con la distribución de los corotipos.

\section{MATERIALES Y MÉTODOS}

La Octava Región de Chile está ubicada en la zona centro sur de Chile ente los $36^{\circ} 00^{\prime}$ y $38^{\circ} 30^{\prime} \mathrm{S}$, abarcando una superficie de 36.930 $\mathrm{km}^{2}$. La región fue dividida con una grilla de $0,25^{\circ} \times 0,25^{\circ}$ de latitud - longitud, fusionando aquellas cuadrículas que presentan menos de la mitad de la superficie de la región con la cuadrícula adyacente (Fig.1).

Se determinó la distribución de 203 especies de árboles y arbustos a partir de la información de la base de datos del Herbario de la Universidad de Concepción (CONC). Esta información fue complementada con datos de la literatura que citan especies para la región, pero cuyas muestras están almacenadas en otros herbarios. Elegimos a los árboles y arbustos dado que las descripciones fitogeográficas hechas para el área han sido realizadas principalmente sobre la base de la distribución de los taxa leñosos, y porque este grupo representa la flora más conspicua de una zona que muchas veces son las principales indicadoras de patrones biogeográficos (IbarraManríquez et al. 2002). La nomenclatura sigue en gran parte a Marticorena \& Quezada (1985).

Sabiendo que existe un gran vacío de colectas especialmente en áreas de difícil acceso que impiden conocer con certeza el rango de distribución de muchas especies se complementaron las distribuciones de varias especies a través del índice de contagio de Araújo \& Williams (2000). Esta medida de contagio asume que la probabilidad de colonización de una especie es dependiente de la densidad de ocurrencias a su alrededor y de las distancias entre aquellas áreas pobladas y el sitio evaluado (Araújo \& Williams 2000). El valor del índice toma el valor uno cuando la cuadrícula evaluada está rodeada por presencias en las ocho cuadrículas que lo rodean (vecinos de primer orden) y en las 16 cuadrículas que rodean a los vecinos de primer orden (vecinos de segundo orden). El índice toma valor cero cuando no existen registros entre los vecinos de primer y segundo orden. La inclusión de una cuadrícula como registro de presencia se realizó en aquellos casos donde el valor del índice superó el tercio superior del total de índices calculados en el mapa, tomando como precaución que el tipo de relieve y rango de altitud de la cuadrícula evaluada fuese similar respecto a las cuadrículas vecinas de primer orden. Para esto último utilizamos una imagen de relieve con cotas de altitud cada 100 metros.

\section{Análisis de clasificación de especies}

Una vez completada la matriz de presencia-ausencia de las 203 especies en las 83 cuadrículas se calculó el índice de similitud Baroni-Urbani $\&$ Buser (1976) a cada par de especies $a$ y $b$ de la matriz.

$$
S=\frac{\sqrt{\mathrm{C} * \mathrm{D}}+\mathrm{C}}{\sqrt{\mathrm{C} * \mathrm{D}}+\mathrm{A}+\mathrm{B}+\mathrm{C}}
$$

donde $\mathrm{A}$ y $\mathrm{B}$ es el número de cuadrículas donde la especie a y b están presentes respectivamente, $\mathrm{C}$ es el número de cuadrículas donde las especies a y b están presente simultáneamente y $\mathrm{D}$ es el número de cuadrículas donde ninguna de las dos especies está presente.

Posteriormente realizamos un análisis de cluster, utilizando el algoritmo UPGMA, donde los grupos obtenidos fueron representados en un dendrograma. Se estimó la significancia de cada nodo mediante la metodología propuesta por McCoy et al. (1986), comenzando por aquellos nodos de menor similitud. Esta metodología consiste en transformar la matriz de similitud en una matriz 
de signos “+”, “-” y "0" de acuerdo a si el valor de similitud entre dos especies es mayor, menor o sin diferencia significativa de lo esperado por azar, respectivamente. Luego se construye una submatriz de signos que solo incluye a las especies del nodo evaluado. En la submatriz definimos tres zonas: zona A, zona B, correspondientes a cada grupo de especies que el nodo separa, y zona A x B correspondiente a la intersección entre A y B (ver McCoy et al. 1986 para mayores detalles). Posteriormente se calcularon los parámetros DW(A x A), DW(B x B) que miden la homogeneidad interna de cada grupo analizado determinando si la proporción de signos “+” tienden a estar concentradas en las zonas A o B pero no en A x B. También se calculó el parámetro DS, que estima si la proporción de similitudes menores que las esperadas por azar (-) tienden a concentrarse en la zona AxB pero no en A ni en B. La significancia estadística de cada nodo fue evaluada mediante una prueba de independencia (prueba de G) de distribución de signos +, - y 0 entre las tres zonas, entregando los parámetros GS para una segregación fuerte y GW para una segregación de tipo débil (ver detalles en McCoy et al. 1986). Un grupo de especies es considerado un corotipo débilmente segregado si GW es significativo y DW > 0, o fuertemente segregado si GS es significativo y DS > 0 (McCoy et al. 1986, Vargas et al. 1997).

El valor de similitud crítica fue calculado mediante un análisis "bootstrap". A partir de la matriz de similitud de especies se generaron en forma aleatoria 1.000 nuevas matrices de similitud. En este conjunto de datos fue calculado el percentil 95 de la distribución, que para nuestro caso representa el valor crítico de similitud con un $95 \%$ de confianza (McCoy et al. 1986).

\section{Variables climáticas}

Con el propósito de relacionar los corotipos con variables ambientales se recopiló información climática de 24 estaciones con más de 15 años de registros continuos dependientes de la Dirección de Aguas del Ministerio de Obras Públicas del Gobierno de Chile, Dirección de Meteorología de Chile y también desde el compendio de Hajek \& Di Castri (1975). Se distribuyen 16 estaciones en la Octava Región, seis al sur y dos al norte. Para cada estación se recopilaron los datos de precipitación media mensual y temperatura media mensual. Sobre la base de las temperaturas y precipitaciones media mensual se definieron 12 nuevas variables climáticas para cada estación con el fin de obtener factores explicatorios que integren los suministros de energía (temperatura) y agua (precipitaciones) al ambiente y con efectos sobre las plantas (Stephenson 1990). Estas variables fueron: temperatura media anual (TAN), temperatura media de invierno (TIN), temperatura media de verano (TVE) y precipitación media anual (PAN), índices ombrotérmicos anuales de Emberger (EMB), De Martone (MAR) y Datin-Revenga (DAT), los índices de continentalidad de Daget (DAG) y de Gorczynski (GOR), el índice de mediterraneidad (MED), índice de aridez (ARI) ambos de RivasMartínez, y la evapotranspiración potencial de Thornwaite (ETP). La determinación de estas variables climáticas se hizo siguiendo lo descrito por Fernández-González (1997).

Se asignó un valor de cada variable climática a las 86 cuadrículas interpolando los datos de las estaciones utilizando el método geoestadístico kriging (Smith et al. 1996). Esta modalidad entrega la tendencia observada en los datos en una imagen de contornos alisada que representa a toda la Octava Región, luego sobreponiendo la grilla de cuadrículas se obtienen los datos requeridos para aquellas cuadrículas sin estaciones climáticas.

El rango de altura (RAN) por cada cuadrícula se obtuvo definiendo una carta de alturas entre las coordenadas $36^{\circ} 00^{\prime}$ y $38^{\circ} 30^{\prime} \mathrm{S}$ y $71^{\circ} 00^{\prime}$ y $74^{\circ} 00^{\prime} \mathrm{O}$ en el programa PanMap (Diepenbroek et al. 2000). Se utilizó la capa GTOPO30 que entrega el vector de elevación cada $100 \mathrm{~m}$ de altura, en esta carta se incluyeron los límites continental, nacional y regional, todos proporcionados por el soporte del software. La grilla de cuadrículas de $0,25^{\circ} \times 0,25^{\circ}$ latitud-longitud fue diseñada de acuerdo al procedimiento indicado por Diepenbroek et al. (2000).

\section{Relación entre corotipos y variables ambientales}

Con el fin de analizar la significancia estadística de la influencia de las variables ambientales en el patrón de distribución de cada uno de los corotipos en las 86 cuadrículas de la Octava Región se utilizó la siguiente regresión logística:

$$
p=\frac{e^{y}}{1+e^{y}}
$$

donde $p$ representa la probabilidad de que el corotipo esté presente, $e$ es la base de los logaritmos neperianos, $y$ es la ecuación de regresión de forma:

$$
y=a+b x_{1}+c x_{2}+\ldots+n x_{n}
$$

donde $x_{\mathrm{n}}$ es una selección de variables ambientales que son incorporadas sucesivamente al 
modelo de regresión mejorando la varianza explicada. El modelo logístico fue evaluado mediante una prueba de bondad de ajuste de $\chi^{2}$ (Vivanco 1999). Este procedimiento permite detectar aquellas variables que explican la presencia de un corotipo en una cuadrícula. La regresión logística analiza la influencia de las variables ambientales en cada corotipo por separado. Por último, identificamos al factor ambiental con más influencia del modelo comparando los "odds ratio" en cada coeficiente de regresión. Con el fin de optimizar la detección de las causas de la distribución de los corotipos, utilizamos en el análisis de regresión aquellas cuadrículas que presentan más del $50 \%$ de las especies que conforman el corotipo (Vivanco 1999). Finalmente, se utilizó un análisis de correspondencia canónica (CCA) para examinar la relación existente entre los corotipos encontrados y las variables ambientales en conjunto (Ter Braak 1986, 1987). Este análisis permite determinar las tendencias de los corotipos en respuesta a las variables ambientales, discriminando patrones de agregaciones por tendencias de distribución común que respondan de forma similar a una o más variables ambientales.

\section{RESULTADOS}

La Fig. 2 muestra el dendrograma obtenido a partir del análisis de agrupamiento, donde se distinguen 24 agrupaciones significativas definidas por las segregaciones fuertes y débiles que se muestran en la Tabla 1. El nombre científico de las 203 especies agrupadas en este estudio se presenta en el Apéndice. La Fig. 3 muestra el patrón de distribución de los 24 corotipos encontrados en la Octava Región, nombrándose las especies representativas de cada uno en la Tabla 2, estas especies corresponden a aquellas que poseen la más amplia distribución dentro del corotipo. De los 24 corotipos, solo 18 presentan tendencias significativas con alguna de las variables climática o geográficas utilizadas en la regresión logística (Tabla 2). Los corotipos que responden significativamente a una o más variables ambientales se describen considerando las especies característica de cada grupo, siendo las especies características aquellas que ocupan más del 50 $\%$ de las cuadrículas del corotipo.

\section{Corotipo I}

Las especies que lo componen tienen un amplio rango de distribución en la región, concentrando la riqueza de especies en la sección central de la costa y parte del valle central (Fig. 3).
Estas son especies típicas de clima mediterráneo con características esclerófilas (Tabla 2), donde la especie con más amplia distribución del grupo es Quillaja saponaria. El principal factor analizado que influye sobre la distribución de este corotipo es la temperatura de invierno (Tabla 2).

\section{Corotipo II}

Estas especies poseen un patrón de distribución amplio, aunque sin un área donde se concentre la riqueza, existiendo varios puntos dispersos sobre la región, donde es posible encontrar más del $75 \%$ de las especies que componen el corotipo (Fig. 3). La especie característica es Baccharis salicifolia. La condición ambiental analizada que principalmente está correlacionada con la presencia de este corotipo es la temperatura de verano, aunque también aportan al modelo, con coeficientes positivos, la temperatura media anual y la aridez (Tabla 2).

\section{Corotipo III}

Las especies de este grupo presentan un patrón de distribución similar al corotipo anterior, en cuanto a las características de las áreas con mayor riqueza de especies. Sin embargo, el rango de distribución del corotipo es más estrecho (Fig. 3). El factor ambiental analizado que mejor está correlacionado con esta distribución es la temperatura media anual, aunque también aportan al modelo la temperatura de verano y la aridez (Tabla 2).

\section{Corotipo IV}

Este grupo de especies posee una amplia distribución presentando sus centros de riqueza en varios puntos de la región, especialmente en la cordillera de la Costa y de los Andes (Fig. 3). La mayoría de sus especies se distribuyen ampliamente en el centro de Chile, siendo la especie más característica del grupo Fabiana imbricata. La distribución de este corotipo está correlacionada con la temperatura de verano (Tabla 2).

\section{Corotipo V}

Las especies de este corotipo se distribuyen principalmente en la zona del clima templado lluvioso y subantártico. En la región, este corotipo se distribuye en la sección sur de la cordillera de la Costa (Nahuelbuta) con algunos representantes en el sur del valle central y precordillera andina (Fig. 3). Tanto la tempera- 


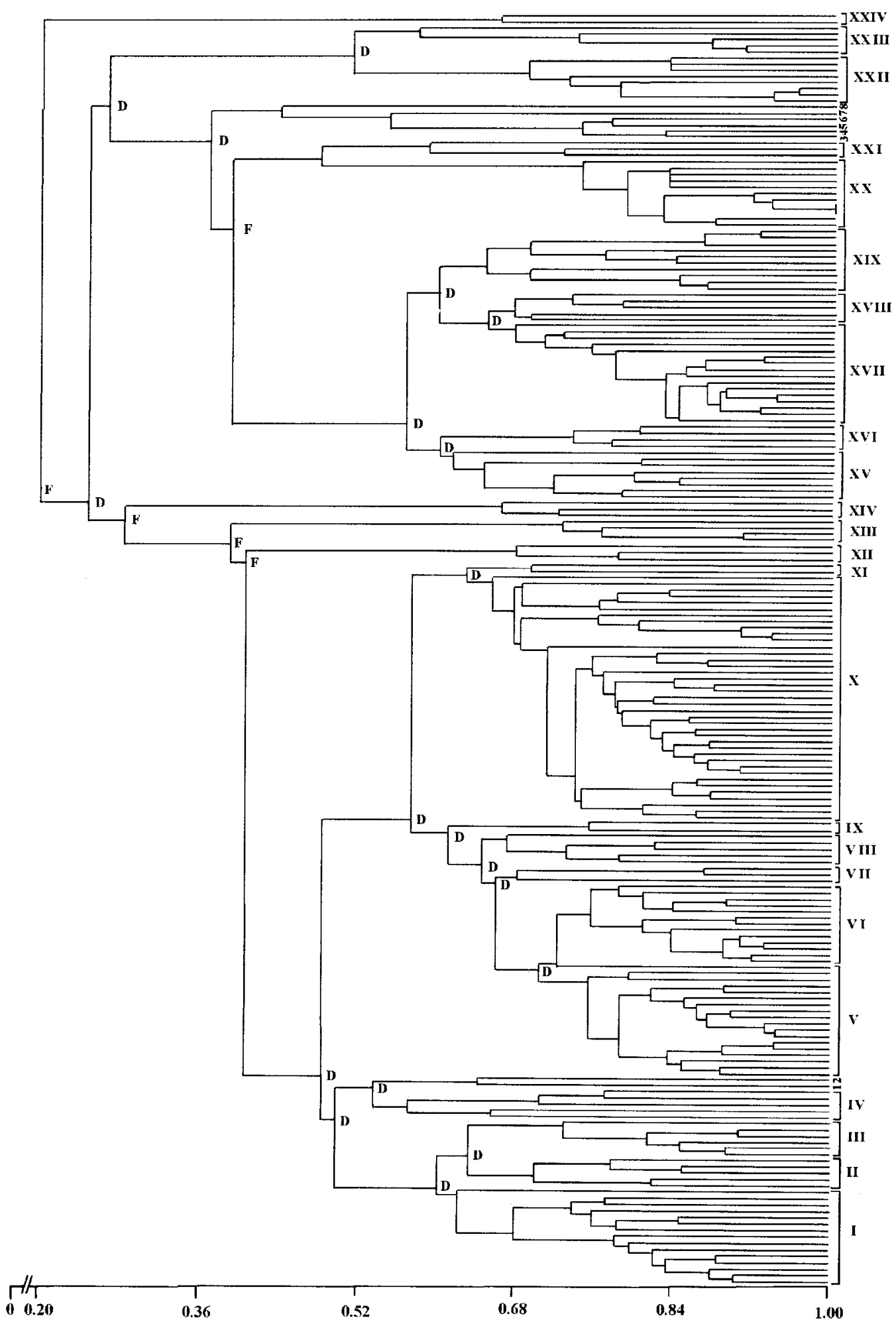

Fig. 2: Dendrograma de similitud obtenido con el índice de Baroni-Urbani \& Buser. F y D indican las segregaciones fuertes y débiles, respectivamente $(\mathrm{P}<0,05)$. Las especies se detallan en el Anexo 1.

Dendrogram obtained with the Baroni-Urbani \& Buser similarity index. Weak (D) and strong (F) segregations (P < 0.05) are indicated. Species are listed in the Appendix 1. 


\section{TABLA 1}

Segregaciones significativas entre los grupos de especies separadas por los nodos del dendrograma. GS y GW indican las segregaciones significativas, DW indica la homogeneidad interna de cada grupo de especies. Para más detalles, ver metodología

Significant segregations between the groups separated by each fork in the dendrogram. GS and GW indicate significant segregations; DW indicates internal homogeneity of each group. For further details, see methods

\begin{tabular}{|c|c|c|c|c|c|c|c|c|}
\hline \multirow[b]{2}{*}{ Nodo } & \multicolumn{3}{|c|}{ UPGMA } & \multicolumn{3}{|c|}{ Segregaciones débiles } & \multicolumn{2}{|c|}{ Segregaciones fuertes } \\
\hline & Grupo A & Grupo B & Coeficiente & $\mathrm{DW}(\mathrm{AxA})$ & DW(BXB) & GW & DS & GS \\
\hline 1 & $1-125$ & $31-181$ & 0,200 & $-0,35$ & 0,68 & 124,7 & 0,08 & 143,0 \\
\hline 2 & $1-114$ & $5-125$ & 0,249 & $-0,04$ & $-0,20$ & 4529,8 & $-0,30$ & 5050,7 \\
\hline 3 & $1-200$ & $44-114$ & 0,284 & 0,02 & 0,69 & 343,5 & 0,26 & 420,3 \\
\hline 4 & $1-202$ & $4-200$ & 0,390 & $-0,06$ & 0,57 & 219,1 & 0,10 & 254,2 \\
\hline 5 & $1-127$ & $38-202$ & 0,405 & $-0,03$ & 0,56 & 166,7 & 0,10 & 202,8 \\
\hline 6 & $5-196$ & $11-125$ & 0,267 & $-0,22$ & 0,14 & 234,5 & $-0,20$ & 264,1 \\
\hline 7 & $5-155$ & $22-196$ & 0,369 & $-0,17$ & $-0,09$ & 111,6 & $-0,24$ & 112,1 \\
\hline 8 & $5-103$ & $7-155$ & 0,391 & 0,14 & 0,28 & 437,7 & 0,10 & 523,1 \\
\hline 9 & $7-140$ & $57-155$ & 0,480 & 0,62 & 0,17 & 84,8 & 0,26 & 74,1 \\
\hline 10 & $1-192$ & $2-127$ & 0,484 & $-0,10$ & 0,09 & 1127,4 & $-0,28$ & 1135,2 \\
\hline 11 & $1-197$ & $21-192$ & 0,498 & 0,20 & $-0,10$ & 141,3 & $-0,15$ & 140,8 \\
\hline 12 & $5-156$ & $6-103$ & 0,567 & 0,02 & 0,07 & 96,6 & $-0,23$ & 90,4 \\
\hline 13 & $21-186$ & $24-192$ & 0,537 & 0,10 & $-0,10$ & 3,7 & $-0,03$ & 5,6 \\
\hline 14 & $2-179$ & $3-127$ & 0,574 & 0,14 & 0,14 & 592,9 & $-0,21$ & 502,9 \\
\hline 15 & $1-133$ & $18-197$ & 0,602 & 0,15 & 0,003 & 45,5 & $-0,31$ & 41,9 \\
\hline 16 & $18-41$ & $30-197$ & 0,633 & 0,10 & 0,10 & 6,8 & $-0,39$ & 8,7 \\
\hline 17 & $2-106$ & $43-179$ & 0,612 & 0,15 & 0,27 & 44,4 & $-0,24$ & 36,6 \\
\hline 18 & $2-165$ & $56-106$ & 0,646 & 0,03 & 0,08 & 29,2 & $-0,51$ & 30,9 \\
\hline 19 & $2-180$ & $27-165$ & 0,660 & 0,09 & 0,08 & 13,6 & $-0,54$ & 13,6 \\
\hline 20 & $2-130$ & $73-180$ & 0,705 & 0,04 & 0,04 & 31,3 & $-0,62$ & 17,1 \\
\hline 21 & $3-143$ & $35-27$ & 0,629 & 0,01 & 0,16 & 13,9 & $-0,48$ & 4,7 \\
\hline 22 & $5-47$ & $45-156$ & 0,601 & 0,14 & 0,27 & 10,1 & $-0,19$ & 10,2 \\
\hline 23 & $6-80$ & $17-103$ & 0,600 & 0,14 & 0,13 & 77,4 & $-0,26$ & 63,2 \\
\hline 24 & $11-144$ & $52-125$ & 0,510 & 0,28 & 0,12 & 23,0 & $-0,20$ & 11,9 \\
\hline 25 & $6-136$ & $34-80$ & 0,650 & 0,03 & 0,04 & 11,8 & $-0,58$ & 10,0 \\
\hline
\end{tabular}

tura de invierno como de verano están correlacionadas con la distribución de este corotipo (Tabla 2).

\section{Corotipo VI}

La mayoría de estas especies tienen una amplia distribución en Chile central, y en la región las especies centran su riqueza a lo largo de la zona costera (Fig. 3). La especie característica es Peumus boldus, y el factor ambiental que mejor está correlacionado con la distribución es la temperatura media de verano y la precipitación anual (Tabla 2).

\section{Corotipo VII}

Los elementos de este corotipo están restringidos a la sección central de la costa de la Octava Región (Fig. 3). La especie característica es $P i$ - 

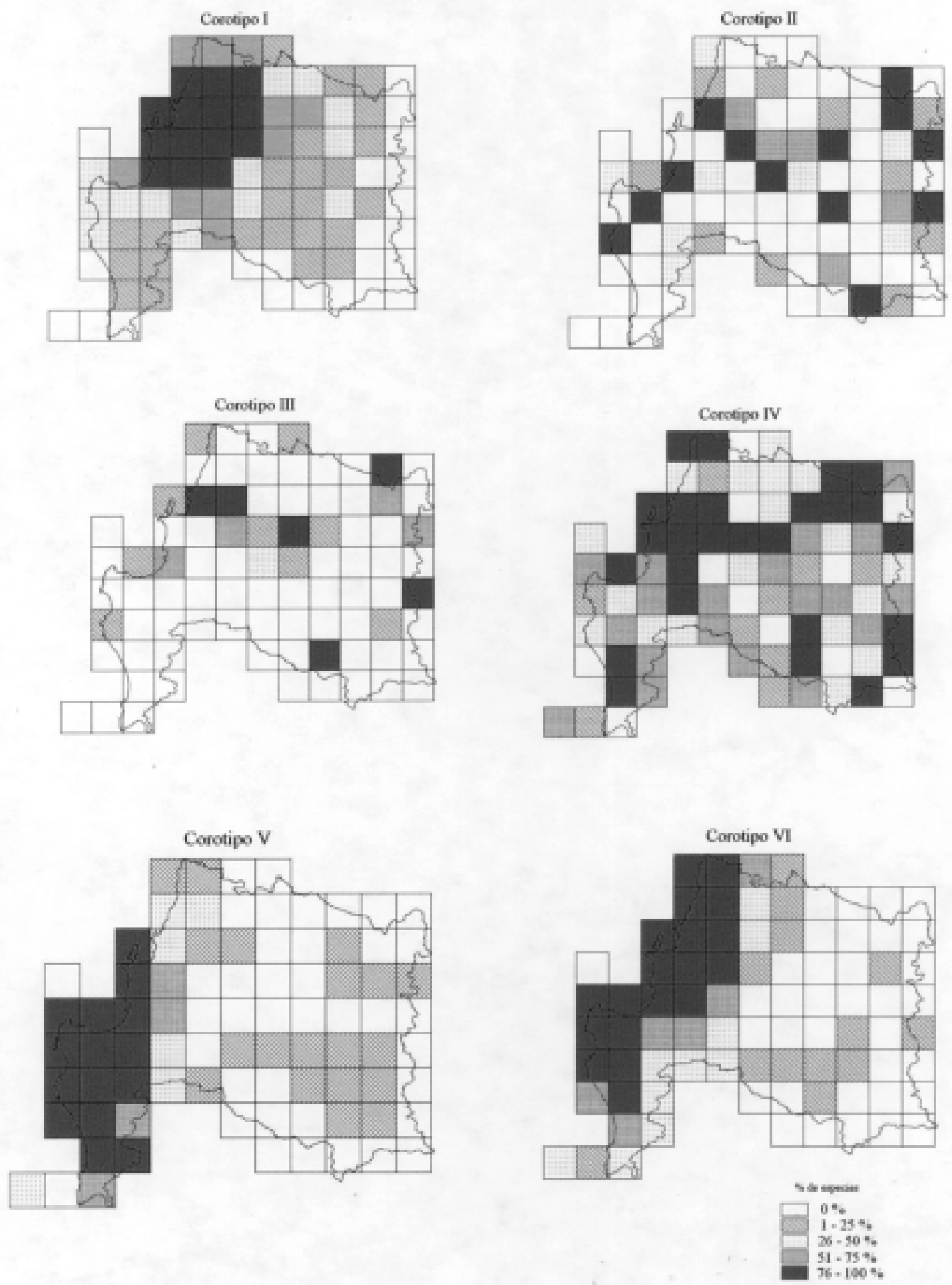

Fig. 3: Mapas de distribución de los 24 corotipos encontrados en la Octava Región de Chile. La leyenda indica el porcentaje de especies de cada corotipo presentes en cada cuadrícula.

Maps with the distribution of the 24 chorotypes found in the Seventh Region of Chile. The percentage of species of each chorotype present at each quadrats is indicated. 

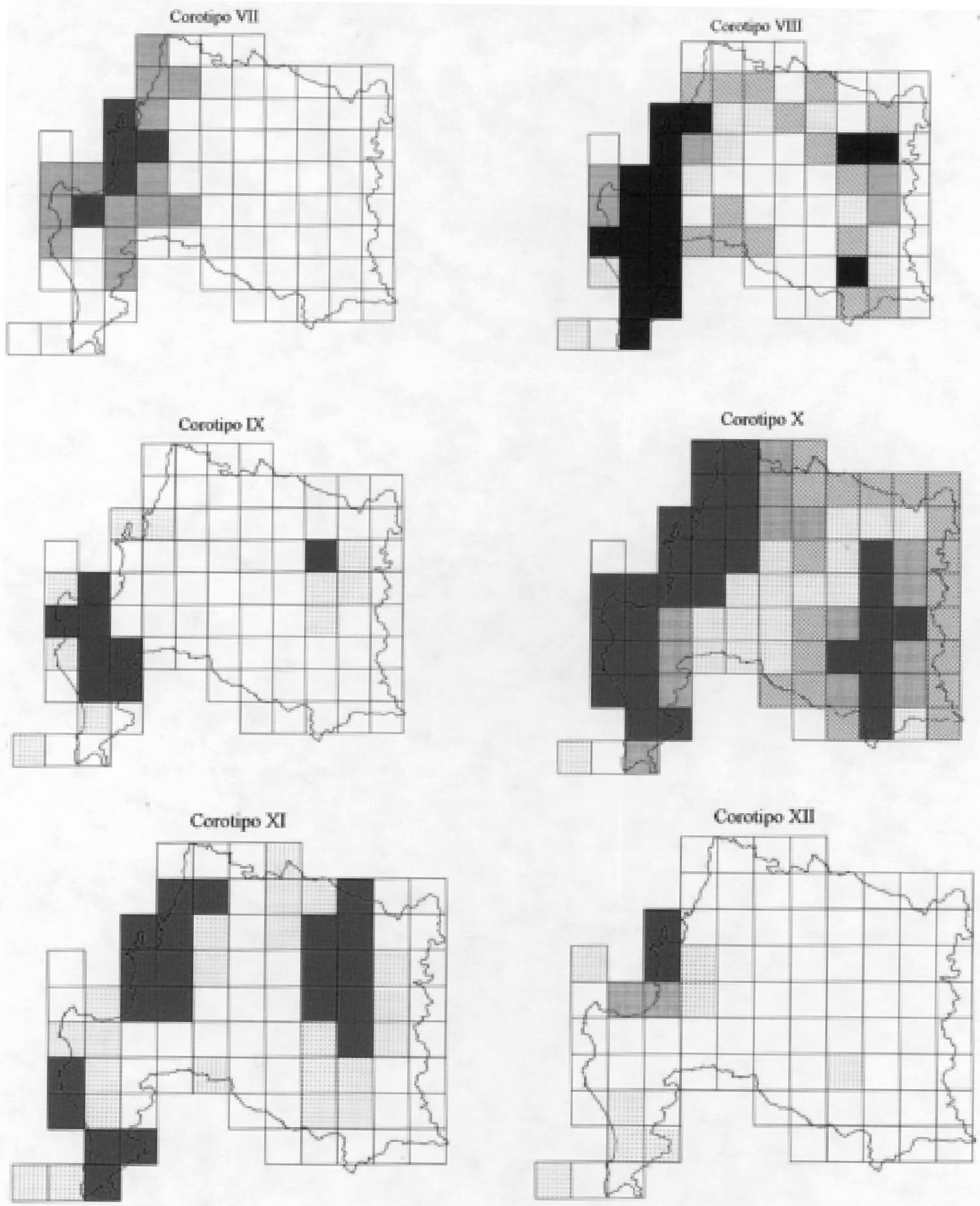

Fig. 3: Continuación.

Continuation. 

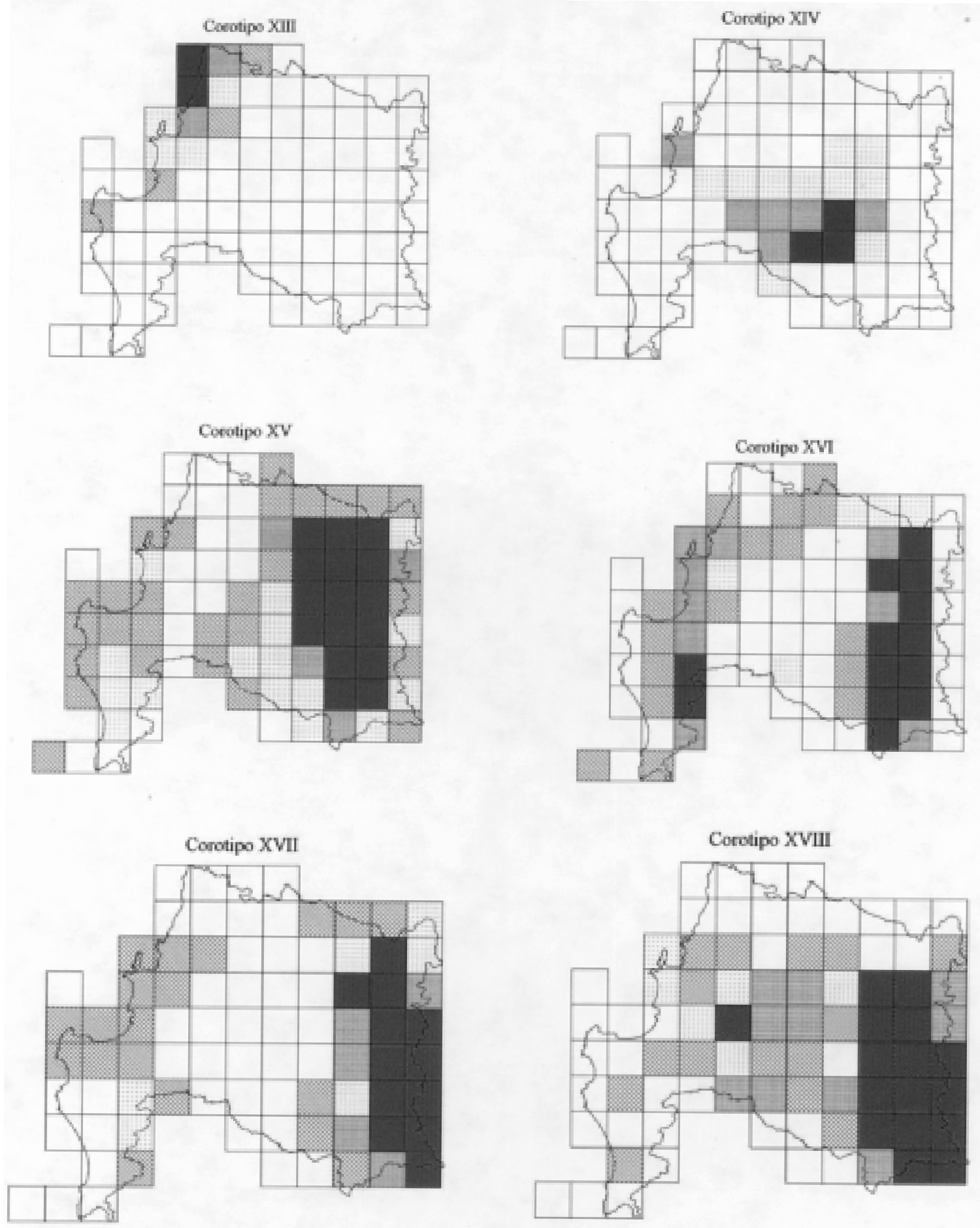

Fig. 3: Continuación.

Continuation. 

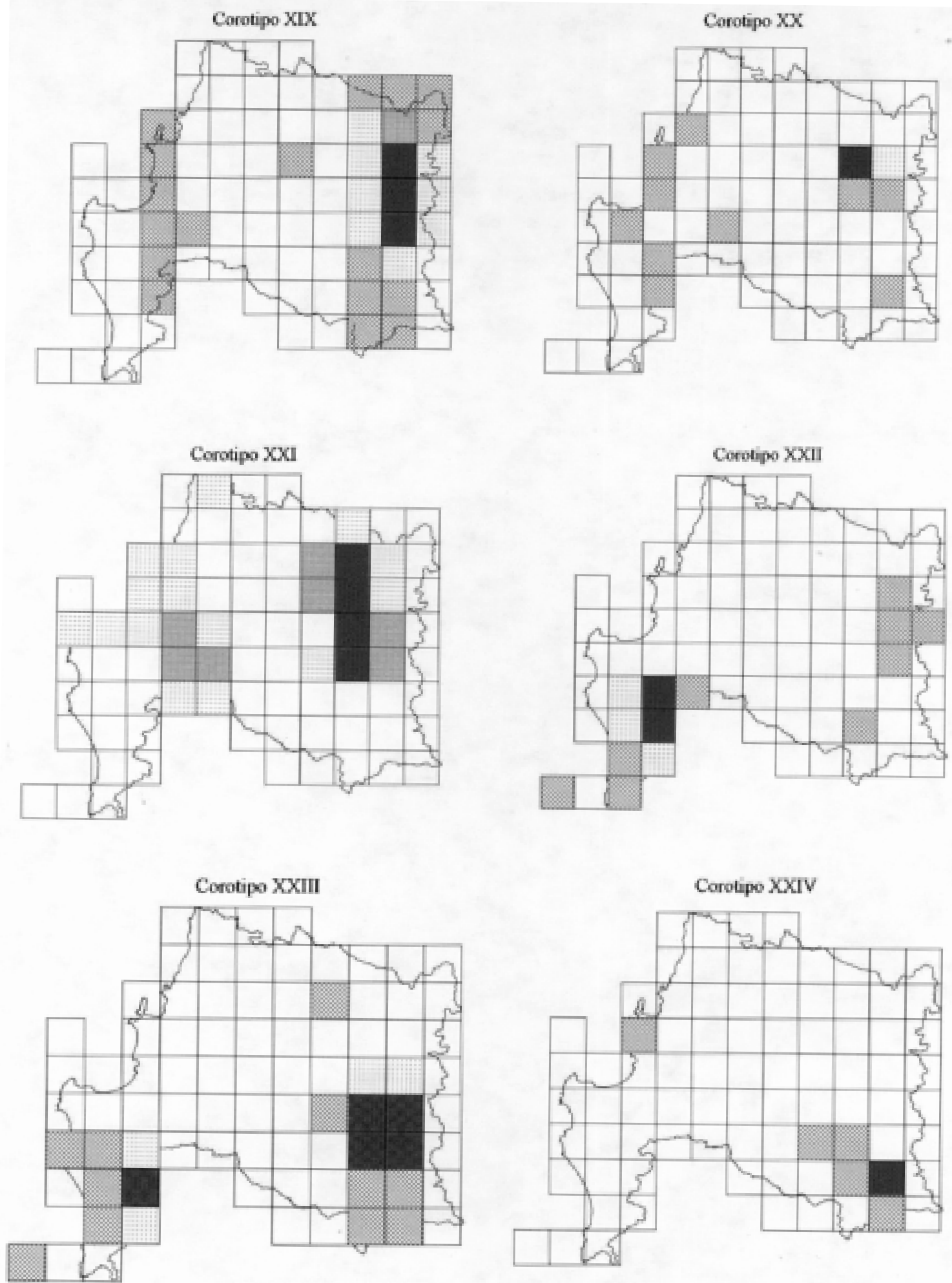

Fig. 3: Continuación.

Continuation. 


\section{TABLA 2}

Especies representativas de los corotipos y ecuaciones de regresión logística indicando para cada corotipo las variables ambientales que aportan en forma significativa al modelo. (*) destaca la variable ambiental con el "odd ratio" mayor

Representative species of each chorotype and logistic regression models with the environmental variables that significantly correlates with the presence of chorotype.

(*) indicates the variable with the highest "odd ratio"

\begin{tabular}{|c|c|c|c|}
\hline Corotipo & Especies & Ecuación & Valor de $\mathrm{P}$ \\
\hline I & $\begin{array}{l}\text { Quillaja saponaria, Luma chequen, Lithrea caustica, } \\
\text { Otholobium glandulosum y Myrceugenia lanceolata }\end{array}$ & $\begin{array}{l}\mathrm{Y}=2,9-2,9 \mathrm{TAN}-1,5 \mathrm{TVE}+4,7 \mathrm{TIN} * \\
+0,5 \mathrm{ARI}+0,8 \mathrm{DAG}+0,4 \mathrm{MED}\end{array}$ & 0,00000 \\
\hline II & $\begin{array}{l}\text { Azara dentata, Haplopappus pectinatus, Baccharis salicifolia, } \\
\text { Haplopappus stolpii y Beilschmiedia berteroana }\end{array}$ & $\mathrm{Y}=-17,1+0,2 \mathrm{TAN}+0,6 \mathrm{TVE}^{*}+0,6 \mathrm{ARI}$ & 0,00005 \\
\hline III & $\begin{array}{l}\text { Baccharis paniculata, Escallonia illinita, Berberis valdiviana, } \\
\text { Berberis negeriana y Herreria stellata }\end{array}$ & $\mathrm{Y}=-12,6+0,5 \mathrm{TAN}^{*}+0,3 \mathrm{TVE}+0,1 \mathrm{ARI}$ & 0,02996 \\
\hline IV & $\begin{array}{l}\text { Azara microphylla, Baccharis obovata, Baccharis neaei, } \\
\text { Fabiana imbricata y Schinus polygama }\end{array}$ & $\begin{array}{l}\mathrm{Y}=1,3+1,0 \text { TVE* }-1,2 \mathrm{TIN}-0,1 \mathrm{ARI}- \\
0,04 \mathrm{DAT}+0,01 \mathrm{MAR}\end{array}$ & 0,00236 \\
\hline $\mathrm{V}$ & $\begin{array}{l}\text { Weinmannia trichosperma, Azara lanceolata, } \\
\text { Caldcluvia paniculata, Myrceugenia leptospermoides y } \\
\text { Sphacele chamaedryoides }\end{array}$ & $\begin{array}{l}\mathrm{Y}=-0,8-1,0 \mathrm{TAN}+2,1 \mathrm{TVE}^{*}+ \\
0,9 \mathrm{TIN}+0,4 \mathrm{ARI}-0,7 \mathrm{DAG}- \\
0,3 \mathrm{DAT}-0,6 \mathrm{MED}-0,9 \mathrm{MAR}\end{array}$ & 0,00000 \\
\hline VI & $\begin{array}{l}\text { Escallonia pulverulenta, Myrceugenia obtusa, } \\
\text { Podanthus ovatifolius, Ugni molinae y Peumus boldus }\end{array}$ & $\begin{array}{l}\mathrm{Y}=12,4-0,002 \mathrm{PAN}+0,02 \mathrm{TVE}^{*} \\
-0,4 \mathrm{DAG}-0,2 \mathrm{DAT}\end{array}$ & 0,00000 \\
\hline VII & Baccharis marginalis, B. sphaerocephala y Pitavia punctata & $\mathrm{Y}=11,2+0,003$ PAN* $-0,5 \mathrm{DAG}-0,27 \mathrm{DAT}$ & 0,00000 \\
\hline VIII & $\begin{array}{l}\text { Buddleja globosa, Embothrium coccineum, Chusquea quila, } \\
\text { Elytropus chilensis y Gaultheria phillyreifolia }\end{array}$ & $\begin{array}{l}\mathrm{Y}=14,8-1,8 \mathrm{TVE}+1,6 \mathrm{TIN} *-0,5 \mathrm{ARI}+ \\
0,002 \text { RAN }\end{array}$ & 0,00000 \\
\hline IX & Berberis actinacantha y Ribes magellanicum & $\mathrm{Y}=-32,1+3,3$ TIN $*-0,8$ ARI $+0,007$ RAN & 0,00005 \\
\hline$X$ & $\begin{array}{l}\text { Aristotelia chilensis, Nothofagus obliqua, Azara integrifolia, } \\
\text { Lomatia hirsuta, Luma apiculata y Drimys winteri }\end{array}$ & $\begin{array}{l}\mathrm{Y}=-2,6+0,8 \text { TAN }-0,5 \text { TVE }-1,1 \text { TIN } \\
+1,4 \text { ARI* }-0,4 \text { DAG + 0,3 MAR }\end{array}$ & 0,00016 \\
\hline XI & Baccharis sagittalis y Leptocarpha rivularis & $\mathrm{Y}=-0,3-0,1 \mathrm{GOR}^{*}$ & 0,03632 \\
\hline XII & Baccharis vernalis, Ercilla volubilis y Vestia foetida & $\mathrm{Y}=-2,2-0,4 \mathrm{DAG}+0,4 \mathrm{MED}^{*}$ & 0,02183 \\
\hline XIII & $\begin{array}{l}\text { Adenopeltis serrata, Bahia ambrosioides, Aristeguietia salvia, } \\
\text { Ugni candollei }\end{array}$ & $Y=-16,8+1,9$ ARI* & 0,02351 \\
\hline XIV & $\begin{array}{l}\text { Berberis chilensis, Haplopappus pedunculosus y } \\
\text { Haplopappus bustillosianus }\end{array}$ & Non significant & \\
\hline $\mathrm{XV}$ & $\begin{array}{l}\text { Adesmia concinna, Eucryphia glutinosa, Hydrangea } \\
\text { serratifolia, Escallonia rubra, y Berberis microphylla }\end{array}$ & $\mathrm{Y}=0,7-0,4 \mathrm{TAN}+0,3 \mathrm{GOR}^{*}$ & 0,00018 \\
\hline XVI & $\begin{array}{l}\text { Berberis darwinii, Myrceugenia ovata, Dasyphyllum } \\
\text { diacanthoides y Nothofagus alpina }\end{array}$ & $\mathrm{Y}=6,2-0,6 \mathrm{TAN}-0,01 \mathrm{ARI} *$ & 0,01740 \\
\hline XVII & $\begin{array}{l}\text { Berberis empetrifolia, Nothofagus pumilio, Nothofagus } \\
\text { antarctica, Escallonia alpina, Austrocedrus chilensis, } \\
\text { Prumnopitys andina }\end{array}$ & $\mathrm{Y}=0,2+1,1 \mathrm{TVE}^{*}-2,2 \mathrm{TIN}$ & 0,00000 \\
\hline XVIII & $\begin{array}{l}\text { Baccharis rhomboidalis, Escallonia virgata, Discaria } \\
\text { articulata, Ephedra chilensis y Discaria trinervis }\end{array}$ & $Y=5,3-0,4$ TVE$^{*}$ & 0,03161 \\
\hline XIX & $\begin{array}{l}\text { Orites myrtoidea, Gaultheria tenuifolia, Diostea juncea, } \\
\text { Viviania ovata, Tetraglochin alatum y Azara celastrina }\end{array}$ & Non significant & \\
\hline $\mathrm{XX}$ & $\begin{array}{l}\text { Calceolaria meyeniana, Ribes cucullatum, Mutisia } \\
\text { ilicifolia, Berberis serratodentata y Solanum ligustrinum }\end{array}$ & Non significant & \\
\hline XXI & Calceolaria alba, Satureja gilliesii y Nothofagus glauca & $Y=-3,6+0,3 \mathrm{DAG}^{*}-0,3 \mathrm{MED}$ & 0,04393 \\
\hline XXII & $\begin{array}{l}\text { Saxegothaea conspicua, Ovidia pillopillo, Empetrum } \\
\text { rubrum, Amomyrtus meli y Campsidium valdivianum }\end{array}$ & Non significant & \\
\hline XXIII & $\begin{array}{l}\text { Berberis trigona, Laureliopsis philippiana, } \\
\text { Maytenus magellanica, Escallonia florida y Ovidia andina }\end{array}$ & Non significant & \\
\hline XXIV & Ribes trilobum y Baccharis patagonica & Non significant & \\
\hline
\end{tabular}


tavia punctata, y la distribución se relaciona principalmente con la precipitación anual y la continentalidad (Tabla 2).

\section{Corotipo VIII}

Las especies de este corotipo poseen una amplia distribución en la Octava Región, situando su centro de riqueza principalmente en la cordillera de la Costa. Sin embargo, también es posible encontrar más del $50 \%$ de las especies de este grupo en dos áreas de la precordillera andina (Fig. 3). El factor ambiental correlacionado con la distribución fue la temperatura de invierno (Tabla 2).

\section{Corotipo IX}

Este grupo está limitado a la zona sur de la región, situando su centro de riqueza de especies principalmente en Nahuelbuta (Fig. 3). La variable climática que se correlaciona a esta distribución fue la temperatura de invierno (Tabla 2).

\section{Corotipo $X$}

Las especies de este corotipo muestran una distribución amplia en la región, ubicándose a lo largo de toda la costa y parte de la cordillera costera el centro de riqueza de especies. También posee centros de riqueza en la sección centro sur de la precordillera andina (Fig. 3). Las especies que caracterizan al conjunto por su notoria distribución son Aristotelia chilensis y Nothofagus obliqua. La aridez es la variable climática que mejor se correlaciona con el corotipo (Tabla 2 ).

\section{Corotipo XI}

El patrón de distribución de estas especies abarca gran parte de la región. Sin embargo, por poseer solo dos taxa su centro de riqueza aparece disperso (Fig. 3). La variable ambiental que explica mejor la distribución de este corotipo es el índice ombrotérmico de Gorczynski (Tabla 2).

\section{Corotipo XII}

Este corotipo presenta una distribución muy restringida en la región (Fig. 3). La especie más conspicua del grupo es Vestia foetida, y la variable ambiental que mejor explica esta distribución es la mediterraneidad (Tabla 2).

\section{Corotipo XIII}

Estas especies se restringen a la zona norte de la costa de la región (Fig. 3). La mayoría de estas especies se distribuyen preferentemente en la costa de Chile central, de ahí que la única variable ambiental utilizada que responde significativamente al modelo de regresión es la aridez, coincidiendo con la formación del secano costero, que en esa zona del país encuentra el límite sur de su distribución (Tabla 2).

\section{Corotipo $X V$}

La distribución de especies de este corotipo abarca la cordillera de los Andes, parte sur del valle central y el sur oeste de la región, con el centro de riqueza de especies en la precordillera andina (Fig. 3). La especie característica es Hydrangea serratifolia. El rango de distribución es amplio para la mayoría de las especies de este corotipo, ubicándose en casi todo Chile incluso en la Patagonia (e.g., Escallonia rubra, Hydrangea serratifolia y Berberis microphylla). El factor ambiental que mejor explica esta distribución es el índice de continentalidad de Gorczynski (Tabla 2).

\section{Corotipo XVI}

Las especies de este corotipo se encuentran principalmente en la zona sur de la condillera de la Costa y de la precordillera andina (Fig. 3). Nothofagus alpina es la especie que destaca en el corotipo, al poseer la más amplia distribución en la región. La variable ambiental que mejor está correlacionada con la distribución de las especies es la aridez (Tabla 2).

\section{Corotipo XVII}

Este grupo presenta un patrón de distribución muy similar al corotipo anterior, la diferencia la marca un centro único de riqueza de especies, ubicado en la precordillera de los Andes (Fig. 3). La especie característica del corotipo es Austrocedrus chilensis. Las temperaturas de invierno y de verano fueron las que mejor se correlacionaron con la distribución, especialmente la temperatura de verano (Tabla 2).

\section{Corotipo XVIII}

Este grupo de especies se distribuye desde la costa a la cordillera de los Andes, sin embargo, es en este último lugar donde se ubica su centro de riqueza (Fig. 3). La principal característica de este corotipo es la gran altitud en que la mayoría de sus especies se desarrollan. Para este corotipo la especie de más amplia distribución es Baccharis rhomboidalis, y la única variable que explica este patrón de distribución es la temperatura de verano (Tabla 2). 


\section{Corotipo XXI}

La distribución de especies de este corotipo se halla repartida entre la zona norte de la precordillera andina y la zona costera. (Fig. 3). La especie que representa al grupo es Satureja gilliesii, y el índice de continentalidad de Daget es quien mejor explica el patrón de distribución observado (Tabla 2).

\section{Análisis de ordenación}

El análisis de correspondencia canónica muestra que los corotipos que presentan sus distribuciones principalmente en la cordillera de los Andes (XXIV, XVII, XIX, XVIII, XV) tienden a dispersarse en el primer cuadrante a lo largo del primer eje de ordenación (Fig. 4). Los corotipos cuyas especies se distribuyen preferentemente en la costa (XXII, IX, V, XII, VII y VI) se dispersan junto al segundo eje de ordenación. Los corotipos I, XIII y XIV que poseen distribuciones muy restringidas o bien muy amplias, escapan al patrón de agrupamiento de corotipos antes explicado. Las correlaciones canónicas intraset muestran que la temperatura de invierno es la variable que presenta la mayor correlación con el eje 1 de ordenación (Tabla 3 ). Para el eje 2 la variable que mejor está correlacionada es la mediterraneidad (Tabla 3).

\section{DISCUSIÓN}

Las técnicas de agrupamiento de especies utilizadas en este trabajo permitieron la obtención de 24 corotipos al interior de la Octava Región de Chile. Esta sería la primera vez que se realiza un análisis de este tipo dentro del país. Estudios similares se han llevado a cabo en otras zonas del mundo, tanto en plantas (e.g., Márquez et al. 1997, Moreno-Sainz et al. 1998) como en animales (e.g., Real et al. 1997, Sans-Fuentes \& Ventura 2000), permitiendo, por un lado, resumir los patrones de distribución de muchas especies a unos pocos patrones comunes (corotipos) $\mathrm{y}$, por otro, evaluar las relaciones entre los corotipos y algunas variables ambientales.

A pesar del alto número de patrones repetitivos encontrados (corotipos) dentro de la Octava Región, los corotipos pueden agruparse en tres patrones generales de distribución. Estos se diferencian de acuerdo a la ubicación de su centro de riqueza de especies en: corotipos de distribución andina, de distribución costera y de amplio rango de distribución, presentando las dos primeras categorías, variantes latitudinales. A su vez, los corotipos encontrados tienen una alta correspondencia con las zonas de vegetación en que ha sido dividida la región (e.g., Gajardo 1994). Por ejemplo, basándonos en la composición de especies reconocemos a

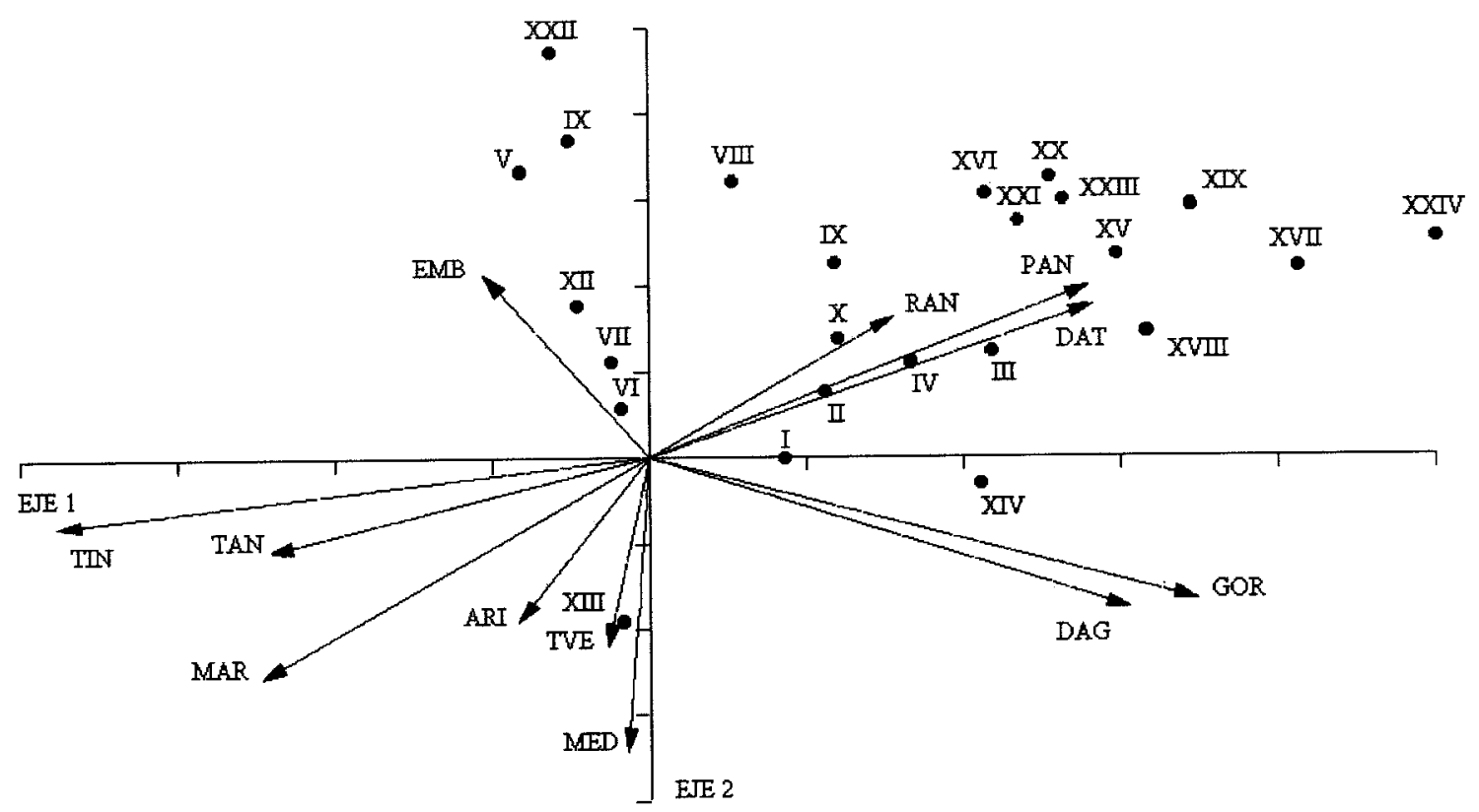

Fig. 4: Análisis de correspondencia canónica (CCA). Los puntos representan a los corotipos, y las flechas señalan la dirección y magnitud del efecto de las variables ambientales sobre los corotipos.

Results of the canonical correspondence analysis (CCA). Points represent the chorotypes, and arrows indicate direction and magnitude of the effect of environmental variables on chorotypes. 
TABLA 3

Correlaciones "intraset" del análisis de correspondencia canónica.

(*) Indica la variable con mejor correlación al eje respectivo

"Intraset" correlations of the canonical correspondence analysis.

(*) Indicates variable with the higher correlation to the respective axis

\begin{tabular}{ccc}
\hline CCA & Eje 1 & Eje 2 \\
\hline Varianza explicada & 0,510 & 0,164 \\
Variable PAN & 0,677 & 0,497 \\
TAN & $-0,582$ & $-0,287$ \\
TVE & $-0,080$ & $-0,509$ \\
TIN & $-0,899^{*}$ & $-0,238$ \\
ARI & $-0,212$ & $-0,451$ \\
DAG & 0,710 & $-0,359$ \\
GOR & 0,811 & $-0,327$ \\
DAT & 0,680 & 0,447 \\
ETP & 0,118 & $-0,124$ \\
MED & $-0,059$ & $-0,786^{*}$ \\
MAR & $-0,605$ & $-0,625$ \\
EMB & $-0,233$ & 0,473 \\
RAN & 0,380 & 0,398 \\
\hline
\end{tabular}

los corotipos I y XIII como representantes del matorral esclerófilo, y en las especies características de esta formación encontramos a Acacia caven, Lithrea caustica, Quillaja saponaria, Adenopeltis serrata y Ugni candollei (Gajardo 1994). En las especies de los corotipos V, VI, X, XXII y XXIII encontramos elementos característicos del bosque siempreverde como las especies Pseudopanax laetevirens, Weinmannia trichosperma, Aextoxicon punctatum, Aristotelia chilensis y Nothogafus dombe$y i$. Por último, encontramos en las especies de los corotipos XVI, XVII, y XXI representantes de la formación de bosques caducifolios ( $\mathrm{Ga}-$ jardo 1994), donde los elementos más representativos son: Nothofagus antarctica, N. pumilio, N. glauca, N. alpina, Prumnopitys andina y Autrocedrus chilensis (Gajardo 1994).

La concordancia entre los corotipos y las características de las especies que los conforman también concuerdan con la zonificación macroclimática de la región (Di Castri \& Hajek 1976). Por ejemplo, los corotipos cuyas especies son representativas del matorral esclerófilo tienden ubicarse en la zona norte de la Octava Región donde predomina el clima tipo mediterráneo, mientras que los corotipos representativos del bosque caducifolio tienden a ubicarse en la zona de la cordillera de los Andes. Los corotipos representantes del bosque siempreverde, probablemente los más extendidos, tienden a ubicarse en la zona centro sur de la Octa- va Región, donde aumentan las precipitaciones y disminuyen las temperaturas estivales.

No obstante, a pesar de identificar estos patrones de distribución en la mayoría de los corotipos existen otros que quedan excluidos de las categorías previas. Entre ellos figuran los corotipos XIV y XX, los cuales, además, no muestran respuesta significativa frente a ninguna variable ambiental analizada. Estos corotipos se ubican en la zona central sur y parte de la precordillera andina. También se agregan a esta condición los corotipos I y XI, que a pesar de haber respondido significativamente a alguna variable ambiental no cumplen con los patrones de distribución general descritos en el párrafo anterior. Estos grupos de especies nos estarían indicando una alta complejidad en la distribución de la flora como consecuencia de la alta heterogeneidad espacial y/o a la importancia de eventos del pasado (e.g., glaciaciones) que otorgan a la flora de esta zona características particulares en cuanto a su distribución, riqueza y endemismos (Arroyo et al. 1995, Villagrán et al. 1995).

Los patrones geográficos de árboles y arbustos revelan que la separación en varios corotipos estaría justificado en la respuesta diferencial que cada corotipo tiene con respecto a las variables ambientales (ver Tabla 4). Nuestros resultados señalan que la temperatura en sus diferentes expresiones sería el factor ambiental que mejor se correlaciona con la distribución de 
la mayoría de los corotipos. Por ejemplo, para los corotipos XVII y XVIII que se distribuyen principalmente en la cordillera, se observaron correlaciones significativas con la temperatura de verano. Esta estación del año tiene una importancia relevante para las plantas de cordillera, ya que es en este periodo en que la vegetación encuentra el momento propicio para el crecimiento y reproducción (Körner 1999).

La temperatura de invierno y su relación con las capacidades de las diferentes especies a tolerar el congelamiento parece ser muy importante, según el análisis de CCA. Por ejemplo, Lomatia hirsuta y Gevuina avellana son especies de la familia Proteaceae que presentan una alta resistencia al frío (Alberdi 1995). Estas especies, pertenecientes al corotipo X, poseen una amplia distribución en la Octava Región, demostrando que su condición de especies frecuentes en los bosques templados puede ser explicado por esta capacidad de soportar las bajas temperaturas en invierno (Alberdi 1995). En esta misma familia se reporta también una alta resistencia al frío en Embothrium coccineum (Alberdi 1995). Sin embargo, esta especie posee una distribución más austral y en la Octava Región se restringe a la zona de la cordillera de la Costa y los Andes, lo cual probablemente esté relacionado con una menor capacidad para tolerar la sequía. Otras especies importantes con relación a resistencia al frío son Nothofagus antarctica y $N$. pumilio, especies que logran soportar hasta $-22{ }^{\circ} \mathrm{C}$ (Alberdi 1987). Diversos autores afirman que el principal factor modelador de la distribución de especies leñosas es la temperatura (Jeffree \& Jeffree 1994) y específicamente las bajas temperaturas de invierno (Sakai \& Weiser 1973, Woodward \& Williams 1987). Para el caso de las especies de los bosques chilenos y su relación con la importancia de la resistencia a las bajas temperaturas cabe destacar el origen de la flora actual de la Octava Región, donde muchos de los taxa que componen la flora actual entre $\operatorname{los} 30^{\circ}$ y $50^{\circ} \mathrm{S}$ son de origen tropical o tiene fuertes relaciones con el reino neotropical (Arroyo et al. 1995, Villagrán \& Hinojosa 1997).

La aridez también está presente en la mayoría de los modelos explicatorios de los corotipos. Por ejemplo, la presencia de los corotipos I, II, III, V y XIII se hallan positivamente relacionados con la aridez (Tabla 4). La distribución de estos corotipos es principalmente hacia la zona norte de la región, manifestando una preferencia con el clima mediterráneo. Con los corotipos IV, VIII, IX y XVI ocurre lo contrario debido al signo negativo que la re- gresión logística asigna a la aridez (Tabla 4). Con ello se indica que la presencia del corotipo se halla desfavorecida bajo condiciones de aridez, los que se distribuyen principalmente hacia la parte sur de la región haciendo notar una preferencia por la influencia algo más húmeda del clima templado. La importancia de la aridez en la distribución de plantas ha sido demostrada en una serie de estudios que han abarcado desde escalas globales (Stephenson 1990, Prentince et al. 1992) hasta escalas regionales (Stephenson 1998).

A pesar de la alta correlación entre los patrones de distribución de especies y diferentes variables macroclimáticas, estamos conscientes que la distribución de especies no puede ser explicada únicamente por factores climáticos. Recientemente Zeilhofer \& Schessl (1999) y Monier \& Abd El-Ghani (2000) han encontrado una fuerte correlación entre la vegetación y las características físicas y químicas del suelo. También, para estos autores poseen mucha importancia los regímenes de perturbaciones como los periodos de inundación y cambios en los regímenes hídricos. Estas últimas variables están, efectivamente, dando cuenta de cambios entre tipos vegetacionales, tanto en estratos arbóreos como en herbáceos (Zeilhofer \& Schessl 1999). Para la flora de Chile central sería esperable que las perturbaciones de gran escala tales como deslizamientos de lava, movimientos de tierra por aluviones, al igual que los tipos de suelos influyan fuertemente sobre los patrones de distribución de especies. Sin embargo, se necesitan más estudios para evaluar su importancia relativa.

La gran cantidad de patrones repetitivos encontrados en este estudio sugiere que los patrones geográficos de distribución de especies al interior de las regiones de Chile son mucho más complejos de lo que usualmente se piensa. Lo anterior tiene importantes implicancias en la comprensión no solo de la historia biogeográfica de nuestra vegetación (Villagrán et al. 1995), también en las posibles respuestas de la flora frente a fenómenos como el calentamiento global del planeta (Arroyo et al. 1993). En efecto, eventos como el descrito anteriormente provocaría variaciones en varios parámetros climáticos, los cuales afectarían de forma particular a cada grupo de taxa. En nuestro estudio encontramos que los diferentes patrones de distribución de especies están afectados por distintos componentes del clima. Por ejemplo, un incremento en las temperaturas afectaría a un grupo determinado de especies, dejando invariante al resto que no presenta sensibilidad a este factor climático. 
Relación significativa entre variables ambientales y presencia de los corotipos según la regresión logística; $(+)$ indica relación positiva, $(-)$ indica una relación negativa entre la presencia de un corotipo y la variable ambiental en cuestión. Clave de abreviaciones: temperatura media anual (TAN), temperatura media invernal (TIN), temperatura media de verano (TVE) y precipitación media anual (PAN); índices ombrotérmicos anuales de Emberger (EMB), De Martone (MAR) y Datin-Revenga (DAT); los índices de continentalidad de Daget (DAG) y de Gorczynski (GOR); el índice de mediterraneidad (MED), índice de aridez (ARI) ambos de Rivas-Martínez, y la evapotranspiración potencial de Thornthwaite (ETP)

Significant relations between environmental variables and chorotype presence according to the logistic regressions; $(+)$ indicates a positive relationship, (-) indicates a negative relationship. Key to abbreviations: annual average temperature (TAN), winter average temperature (TIN), summer average temperature (TVE) and total annual rainfall (PAN); Annual ombrothermics index of Emberger (EMB), De Martone (MAR) and Datin-Revenga (DAT), continentality index of Daget (DAG) and Gorczynski (GOR); Rivas-Martínez's mediterreanity index (MED) and aridity index (ARI) and potential evapotranspiration of Thornthwaite (ETP)

\begin{tabular}{|c|c|c|c|c|c|c|c|c|c|c|c|c|c|}
\hline \multirow[t]{2}{*}{ Corotipo } & \multicolumn{13}{|c|}{ Variable ambiental } \\
\hline & TAN & TIN & TVE & PAN & ARI & DAG & GOR & DAT & ETP & MED & MAR & EMB & RAN \\
\hline I & - & + & - & & + & + & & & & + & & & \\
\hline II & + & & + & & + & & & & & & & & \\
\hline III & + & & + & & + & & & & & & & & \\
\hline IV & & - & + & & - & & & - & & & + & & \\
\hline V & - & + & + & & + & - & & - & & - & - & & \\
\hline VI & & & + & - & & - & & - & & & & & \\
\hline VII & & & & + & & - & & - & & & & & \\
\hline VIII & & + & - & & - & & & & & & & & + \\
\hline IX & & + & & & - & & & & & & & & + \\
\hline $\mathrm{X}$ & + & - & - & & + & - & & & & & + & & \\
\hline XI & & & & & & & - & & & & & & \\
\hline XII & & & & & & - & & & & + & & & \\
\hline XIII & & & & & + & & & & & & & & \\
\hline$X V$ & - & & & & & & + & & & & & & \\
\hline XVI & - & & & & - & & & & & & & & \\
\hline XVII & & & + & & & & & & & & & & \\
\hline XVIII & & & - & & & & & & & & & & \\
\hline XXI & & & & & & + & & & & - & & & \\
\hline
\end{tabular}

Finalmente, los corotipos aquí definidos presentan características propias de los dos tipos climáticos presentes en la Octava Región de Chile, el tipo mediterráneo y el tipo templado. Por lo tanto, los análisis utilizados en este trabajo nos permiten apoyar la hipótesis de una transición bioclimática en la Octava Región representada en la flora arbórea y arbustiva, estableciéndose que sería la tolerancia a las bajas temperatura y a la aridez los factores ambiental estudiados que mejor explican los patrones de distribución geográficos.

\section{AGRADECIMIENTOS}

Los autores desean agradecer al Dr. Clodomiro Marticorena, Dr. Roberto Rodríguez, Srta. Maritza Mihoc y Srta. Alejandra Jiménez por ayuda en la recolección y análisis de los datos. E.T. agradece a la beca de Magíster del Núcleo Milenio, P99-103 F ICM - Center of Advances Studies in Ecology and Research in Biodiversity. Investigación financiada por el proyecto FONDECYT 1000364. 


\section{LITERATURA CITADA}

ALBERDI M (1987) Ecofisiología de las especies chilenas del género Nothofagus. Bosque (Chile) 8: 77-84.

ALBERDI M (1995) Ecofisiología de las especies leñosas de los bosques higrófilos templados de Chile: resistencia a la sequía y a bajas temperaturas. En: Armesto J, C Villagrán \& MK Arroyo (eds) Ecología de los bosques nativos de Chile: 279-300. Editorial Universitaria, Santiago, Chile.

ARAÚJO M \& P WILLIAMS (2000) Selecting areas for species persistence using occurrence data. Biological Conservation 96: 331-245.

ARROYO MTK, J ARMESTO, F SQUEO \& J GUTIÉRREZ (1993) Global change: the flora and vegetation of Chile. En: Mooney H, E Fuentes \& BI Kronberg (eds) Earth system response to global change: contrast between North and South America: 239-263. Academic Press, San Diego, California, USA.

ARROYO MTK, LA CAVIERES, A PEÑALOZA, M RIVEROS \& AM FAGGI (1995) Relaciones fitogeográficas y patrones regionales de riqueza en la flora del bosque lluvioso templado del sur de Sudamérica. En: Armesto JJ, C Villagrán \& MTK Arroyo (eds) Ecología de los bosques nativos de Chile: 71-100. Editorial Universitaria, Santiago, Chile.

BARONI-URBANI C \& M BUSER (1976) Similarity of binary data. Systematic Zoology 25: 251-259.

BIRKS H J B (1987) Recent methodological developments in quantitative descriptive biogeography. Annali Zoologi Fennici 24: 165-178.

BLASI C, ML CARRANZA, L FILESI, A TILIA \& A ACOSTA (1999) Relation between climate and vegetation along a Mediterranean-temperate boundary in central Italy. Global Ecology \& Biogeography 8: 17-27.

BROWN JH \& MV LOMOLINO (1998) Biogeography. Second edition. Sinauer Associates, Sunderland, Massachusetts, USA. xxi-692 pp.

CAVIERES LA, M MIHOC, A MARTICORENA, C MARTICORENA, M BAEZA, O MATTHEI \& MTK ARROYO (2004) La flora de la cordillera de la Costa en la Región del Biobío: patrones de distribución de riqueza de especies, géneros familias y endemismos. En: Smith-Ramírez C, JJ Armesto \& C Valdovinos (eds) Ecología y biodiversidad de la cordillera de Costa de Chile. Editorial Universitaria (en prensa).

DI CASTRI F \& E HAJEK (1976) Bioclimatología de Chile. Ediciones de la Pontificia Universidad Católica de Chile, Santiago, Chile. 170 pp.

DIEPENBROEK M, H GROBE \& R SIEGER. (2000) PanMap, www.pangea/software/panmap

FERNÁNDEZ-GONZÁLEZ F (1997) Bioclimatología. En: Izco J, Barreno E, Brugués M, Costa M, Devesa J, Fernández F, Gallardo T, Llimona X, Salvo E, Talavera S \& B Valdés (eds) Botánica. McGrawHill, Interamericana de España, Madrid, España.

GAJARDO R (1994) La vegetación natural de Chile. Clasificación y distribución geográfica. Editorial Universitaria, Santiago, Chile. $314 \mathrm{pp}$.

HAJEK E \& F DI CASTRI (1975) Bioclimatografía de Chile. Dirección de Investigación, Universidad Católica de Chile, Santiago, Chile. 223 pp.

HUBER A (1975) Beitrag zur klimatologie und klimaökologie von Chile. Inaugural-dissertation zur Erlangung der Doktorwürde des facbereinchs forstwirtschaft der Ludwig Maximilians-Universität zu Munchen, Munchen, Germany. 87 pp.
IBARRA-MANRÍQUEZ G, JL VILLASEÑOR, R DURÁN \& J MEAVE (2002) Biogeographycal analysis of the tree flora of Yucatán Peninsula. Journal of Biogeography 29: 17-29.

JEFFREE E \& C JEFFREE (1994) Temperature and the biogeographical distribution of species. Functional Ecology 8: 640-650.

KÖRNER C (1999) Alpine plant life. Springer-Verlag, Berlin, Germany. 338 pp.

MARAÑON T, R AJBILOU, F OJEDA \& J ARROYO (1999) Biodiversity of woody species in oak woodlands of southern Spain and northern Morocco. Forest Ecology and Management 115: 147-156

MARTICORENA C \& M QUEZADA (1985) Catálogo de la flora vascular de Chile. Gayana Botánica (Chile) 42: 5-155.

MÁRQUEZ A, R REAL, J VARGAS \& A SALVO (1997) On identifying common distribution pattern and their causal factor: a probabilistic method applied to Pteridophytes in the Iberian Peninsula. Journal of Biogeography 24: 613-631.

McCOY E, S BELL \& K WALTERS (1986) Identifying boundaries along environmental gradient. Ecology 67: 749-759.

MONIER M \& M ABD EL-GHANI (2000) Floristic and environmental relations in two extreme desert zones of western Egypt. Global Ecology and Biogeography 9: 449-516.

MORENO-SAIZ JC, I CASTRO PARGA \& H SAINZ OLLERO (1998) Numerical analyses of distribution of Iberian and Balearic monocotyledons. Journal of Biogeography 25: 179-194.

MORLA C \& F PINEDA (1985) The woody vegetation in the Mediterranean-atlantic boundary in the north-west of the Iberian Peninsula. Candollea 40: 435-446.

OBERDORFER E (1960) Pflanzensoziologische Studien in Chile, Ein Vergleich mit Europa. Flora et Vegetatio Mundi 2: 1-128

PISANO E (1956) Esquema de clasificación de las comunidades vegetales de Chile. Agronomía (Chile) 2: 30-33.

PRENTINCE IC, W CRAMER, SP HARRISON, R LEEMANS RA MONSERUD \& AM SOLOMON (1992) A global biome model based on plant physiology and dominance, soil properties and climate. Journal of Biogeography 19: 117-134.

REAL R, J GUERRERO \& J RAMIREZ (1992a) Identificación de fronteras bióticas significativas para los anfibios de la cuenca hidrográfica del sur de España. Doñana Acta Vertebrata 19: 53-70.

REAL R, J VARGAS \& J GUERRERO (1992b) Análisis biogeográfico de clasificación de áreas y especies. Monographs in Herpetology 2: 73-84

REAL R, J PLEGUEZUELOS \& S FAHD (1997) The distribution pattern of reptiles in the Riff region, northern Morocco. African Journal of Ecology 35: 312- 325.

SAKAI A \& C WAISER (1973) Freezing resistance of trees in North America with reference to three regions. Ecology 54: 118-125.

SANS-FUENTES MA \& J VENTURA (2001) Distribution patterns of the small mammals (Insectivora and Rodentia) in a transitional zone between the Eurosiberian and the Mediterranean regions. Journal of Biogeography 27: 755-764.

SCHMITHÜSEN J (1956) Forschungen in Chile. Die räumliche Ordnung der chilenischen Vegetation. Bunner Geographische Abhandlungen 17: 1-86.

SMITH D, W WALL, Z CHEN, R BARNES \& B SIMONS (1996) Surfer (Win 32) Version 6.04 Surface 
Mapping System., Golden Software, Inc., Boulder, Colorado, USA.

STEPHENSON N (1990) Climatic control of vegetation distribution: the role of the water balance. American Naturalist 135: 649-670.

STEPHENSON N (1998) Actual evapotranspiration and deficit: biological meaningful correlations of vegetation distribution across spatial scales. Journal of Biogeography 25: 855-870.

TER BRAAK C (1986) Canonical correspondence analysis: a new eigenvector technique for a multivariate direct gradient analysis. Ecology 67: 1167-1179.

TER BRAAK C (1987) The analysis of vegetation environmental relationship by canonical correspondence analysis. Vegetatio 69: 69-77.

VARGAS J, R REAL \& L PALOMO (1997) On identifying significant co-occurrence of species in space and time. Miscellania Zoologica 20: 49-58.
VILLAGRÁN C \& L HINOJOSA (1997) Historia de los bosques templados del sur de Sudamérica, II: análisis fitogeográfico. Revista Chilena de Historia Natural 70: 241-267.

VILLAGRÁN C, P MORENO \& R VILLA (1995) Antecedentes palinológicos acerca de la historia cuaternaria de los bosques chilenos. En: Armesto J, C Villagrán \& MK Arroyo (eds.) Ecología de los bosques nativos de Chile: 51-65. Editorial Universitaria, Santiago, Chile.

VIVANCO M (1999) Análisis estadístico multivariable: teoría y práctica. Editorial Universitaria, Santiago, Chile. 223 pp.

WOODWARD F I \& F G WILLIAMS (1987) Climate and plant distribution at global and local scales. Vegetatio 69: 189-197.

ZEILHOFER P \& M SCHESSL (1999) Relationship between vegetation and environmental conditions in the northern Pantanal of Mato Grosso, Brazil. Journal of Biogeography 27: 159-168

Editor Asociado: Javier Figueroa

Recibido el 14 de marzo de 2003; aceptado el 10 de octubre de 2003 


\section{ANEXO 1}

Composición específica de cada corotipo. Se incluyen las especies que no han conformado grupos significativos

Specific composition for each chorotype. Species that do not belong to statistically significant groups are included

Grupos no significativos

1 Baccharis linearis (Ruiz et Pav.) Pers.

2 Sophora cassioides (Phil.) Sparre

3 Azara petiolaris (D. Don) I.M. Johnst.

Escallonia myrtoidea Bertero ex DC.

5 Legrandia concinna (Phil.) Kausel

6 Mutisia linearifolia Cav.

7 Mutisia latifolia D. Don

8 Tepualia stipularis (Hook. et Arn.) Griseb.

Corotipo I

Acacia caven (Molina) Molina

Ageratina glechonophylla (Less.) R.M.King et H.Rob.

Colliguaja dombeyana A.Juss.

Kageneckia oblonga Ruiz et Pav.

Teucrium bicolor $\mathrm{Sm}$.

Retanilla ephedra (Vent.) Brongn.

Gochnatia foliolosa (D.Don) D.Don ex Hook et Arn.

Salix humboldtiana Willd.

Cestrum parqui L'Hér.

Lithrea caustica (Molina) Hook. et Arn.

Otholobium glandulosum (L.) J.W.Grimes

Escallonia revoluta (Ruiz et Pav.) Pres.

Myrceugenia lanceolata (Juss. ex J.St.-Hil.) Kausel

Quillaja saponaria Molina

Luma chequen (Molina) A.Gray

Corotipo II

Azara dentata Ruiz et Pav.

Haplopappus pectinatus Phil.

Baccharis salicifolia (Ruiz et Pav.) Pers.

Haplopappus stolpii Phil.

Beilschmiedia berteroana (Gay) Kosterm.

Corotipo III

Baccharis paniculata DC.

Escallonia illinita K.Presl

Berberis valdiviana Phil.

Berberis negeriana Tischler

Herreria stellata Ruiz et Pav.

Tessaria absinthioides (Hook. et Arn.) DC.

Corotipo IV

Azara microphylla Hook.f.

Baccharis obovata Hook. et Arn.

Baccharis neaei DC.

Fabiana imbricata Ruiz et Pav.

Schinus polygama (Cav.) Cabrera

Corotipo V

Acrisione cymosa (J.Remy) B.Nord.

Rhamnus diffusus Clos

Corynabutilon vitifolium (Cav.) Kearney

Chusquea valdiviensis E.Desv.

Pseudopanax laetevirens (Gay) Franchet

Pseudopanax valdiviensis (Gay) Seem. ex Reiche

Amomyrtus luma (Molina) D.Legrand et Kausel

Eucryphia cordifolia Cav.

Escallonia leucantha J.Remy

Berberidopsis corallina Hook.f.

Weinmannia trichosperma Cav.

Azara lanceolata Hook.f.
Caldcluvia paniculata (Cav.) D.Don

Myrceugenia leptospermoides (DC.) Kausel

Sphacele chamaedryoides (Balbis) Briq.

Desfontainia spinosa Ruiz et Pav.

Lomatia ferruginea (Cav.) R.Br.

Corotipo VI

Crinodendron patagua Molina

Escallonia pulverulenta (Ruiz et Pav.) Pers.

Myrceugenia obtusa (DC.) O.Berg

Podanthus ovatifolius Lag.

Ugni molinae Turcz.

Senna stipulacea (Aiton) H.S. Irwin et Barneby

Peumus boldus Molina

Gaultheria insana (Molina) D.J. Middleton

Griselinia jodinifolia (Griseb.) Taubert

Gomortega keule (Molina) Baillon

Griselinia scandens (Ruiz. et Pav.) Taubert

Myrceugenia planipes (Hook. et Arn.) O.Berg

Myrceugenia pinifolia (F.Phil.) Kausel

Ribes punctatum Ruiz et Pav.

Corotipo VII

Baccharis marginalis DC.

Baccharis sphaerocephala Hook. et Arn.

Pitavia punctata (Ruiz et Pav.) Molina

Corotipo VIII

Buddleja globosa Hope

Embothrium coccineum J.R. Forst. et G. Forst.

Chusquea quila Kunth

Elytropus chilensis (A.DC.) Müll. Arg.

Gaultheria phillyreifolia (Pers.) Sleumer

Corotipo IX

Berberis actinacantha Mart.

Ribes magellanicum Poir.

Corotipo X

Acrisione denticulata (Hook. et Arn.) B. Nord.

Myrceugenia parvifolia (DC.) Kausel

Citronella mucronata (Ruiz et Pav.) D.Don

Baccharis racemosa (Ruiz et Pav.) DC.

Solanum crispum Ruiz et Pav.

Gaultheria mucronata (L.f.) Hook. et Arn.

Mitraria coccinea Cav.

Aextoxicon punctatum Ruiz et Pav.

Lapageria rosea Ruiz et Pav.

Lomatia dentata (Ruiz et Pav.) R. Br.

Nothofagus dombeyi (Mirb.) Oerst.

Lardizabala biternata Ruiz et Pav.

Proustia pyrifolia DC.

Muehlenbeckia hastulata (Sm.) I.M.Johnst.

Podocarpus saligna D.Don

Cissus striata Ruiz et Pav.

Drimys winteri J.R.Forst. et G. Forst

Cryptocarya alba (Molina) Looser

Gevuina avellana Molina

Rhaphithamnus spinosus (Juss.) Moldenke

Blepharocalyx cruckshanksii (Hook. et Arn.) Niedenzu

Persea lingue Nees

Boquila trifoliolata (DC.) Decne

Laurelia sempervirens (Ruiz et Pav.) Tul. 
Fuchsia magellanica Lam.

Lomatia hirsuta (Lam.) Diels ex J.F. Macbr. Nothofagus obliqua (Mirb.) Oerst.

Coriaria ruscifolia $\mathrm{L}$.

Aristotelia chilensis (Molina) Stuntz

Azara integrifolia Ruiz et Pav.

Luma apiculata (DC.) Burret

Colletia hystrix Clos

Myrceugenia exsucca (DC.) O.Berg

Azara serrata Ruiz et Pav.

Calceolaria dentata Ruiz et Pav.

Maytenus boaria Molina

Sophora macrocarpa $\mathrm{Sm}$

Discaria chacaye (G. Don) Tortosa

Myoschilos oblonga Ruiz et Pav.

Corotipo XI

Baccharis sagittalis (Less.) DC.

Leptocarpha rivularis DC.

Corotipo XII

Baccharis vernalis F.H. Hellwig

Ercilla volubilis A. Juss.

Vestia foetida (Ruiz et Pav.) Hoffmanns.

Corotipo XIII

Adenopeltis serrata (W.T.Aiton) I.M. Johnst.

Bahia ambrosioides Lag.

Aristeguietia salvia (Colla) R.M. King et H. Rob.

Ugni candollei (Barnéoud) O. Berg

Corotipo XIV

Berberis chilensis Gillies ex Hook. et Arn.

Haplopappus pedunculosus J. Remy

Haplopappus bustillosianus J. Remy

Corotipo XV

Adesmia concinna Phil.

Eucryphia glutinosa (Poepp. et Endl.) Baillon

Azara alpina Poepp. et Endl.

Proustia cuneifolia D. Don

Chusquea culeou E. Desv.

Escallonia rubra (Ruiz et Pav.) Pers.

Hydrangea serratifolia (Hook. et Arn) F. Phil.

Berberis microphylla $\mathrm{G}$. Forst.

Corotipo XVI

Berberis darwinii Hook.

Myrceugenia ovata (Hook. et Arn.) O. Berg

Dasyphyllum diacanthoides (Less.) Cabrera

Nothofagus alpina (Poepp. et Endl.) Oerst.

Corotipo XVII

Adesmia emarginata $\mathrm{Clos}$

Berberis montana Gay

Nothofagus antarctica (G. Forst.) Oerst.

Berberis rotundifolia Poepp. et Endl.

Chiliotrichum rosmarinifolium Less.

Nothofagus pumilio (Poepp. et Endl.) Krasser

Escallonia alpina Poepp. ex DC.

Austrocedrus chilensis (D.Don) Pic.Serm. et Bizzarri

Baccharis magellanica (Lam.) Pers.

Berberis empetrifolia Lam.

Prumnopitys andina (Poepp. et Endl.) De Laub.
Maytenus chubutensis (Speg.) Lourteig, O`Donell

et Sleumer

Gaultheria poeppigii DC.

Araucaria araucana (Molina) K. Koch

Schinus patagonica (Phil.) I.M. Johnst.

Maytenus disticha (Hook.f.) Urb.

Corotipo XVIII

Baccharis rhomboidalis J. Remy

Escallonia virgata (Ruiz et Pav.) Pers.

Discaria articulata (Phil.) Miers

Ephedra chilensis K. Presl.

Discaria trinervis (Gillies ex Hook. et Arn.) Reiche

Corotipo XIX

Azara celastrina D.Don

Baccharis poeppigiana DC.

Tetraglochin alatum (Gillies ex Hook. et Arn.) Kuntze

Viviania ovata Phil

Diostea juncea Miers.(Gillies et Hook.) Miers

Orites myrtoidea (Poepp. et Endl.) Benth. et Hook.f.

ex B.D. Jacks.

Gaultheria tenuifolia (Phil.) Sleumer

Escallonia gayana Acevedo et Kausel

Escallonia rosea Griseb.

Gaultheria caespitosa Poepp. et Endl.

Corotipo XX

Adesmia propinqua $\mathrm{Clos}$

Calceolaria meyeniana Phil.

Ribes bicolor Phil.

Solanum cyrtopodium Dunal

Ribes cucullatum Hook. et Arn.

Solanum ligustrinum Lodd.

Baccharis lycioides J. Remy

Berberis serratodentata Lechler

Haplopappus chrysanthemifolius (Less.) DC.

Solanum valdiviense Dunal

Mutisia ilicifolia Cav.

Corotipo XXI

Calceolaria alba Ruiz et Pav.

Satureja gilliesii Benth.

Nothofagus glauca (Phil.) Krasser

Corotipo XXII

Amomyrtus meli (Phil.) D. Legrand et Krausel

Campsidium valdivianum (Phil.) Skottsb.

Saxegothaea conspicua Lindl.

Ovidia pillopillo (Gay) Meisn.

Empetrum rubrum Vahl ex Willd.

Baccharis zoellneri F.H. Hellwig

Drimys andina (Reiche) R.A. Rodr. et Quezada

Myrceugenia chrysocarpa (O. Berg) Kausel

Corotipo XXIII

Berberis trigona Kunze ex Poepp. et Endl.

Escallonia florida Poepp. ex DC.

Ovidia andina (Poepp. et Endl.) Meisn.

Maytenus magellanica (Lam.) Hook.f.

Laureliopsis philippiana (Looser) Schodde

Corotipo XIV

Baccharis patagonica Hook. et Arn.

Ribes trilobum Meyen 